\title{
Amplitude and Phase Shaping of Ultra-broad- bandwidth Femtosecond Laser Pulses
}

\author{
Bingwei Xu, Yves Coello, D. Ahmasi Harris, Vadim V. Lozovoy, and Marcos \\ Dantus
}

Michigan State University, Department of Chemistry, East Lansing, Michigan 48824 dantus@msu,edu

\begin{abstract}
We report amplitude and phase shaping of an ultra-broad-bandwidth (400 nm) femtosecond laser using a grating-based pulse shaper without precompression. We demonstrate phase compensation with deviation within 0.1 rad and accurate binary phase shaping.
\end{abstract}

\section{Introduction}

Motivations for the use of shaped ultra-broad-bandwidth pulses are selective microenvironment probing, multiphoton microscopy, functional imaging and chemical agent identification. In a recent work, Binhammer et al.[1] produced 4.3 fs pulses, the shortest pulses generated directly from a laser oscillator. This work shows the possibilities of using shaped ultra-broad-bandwidth pulses (spanning over $400 \mathrm{~nm}$ ) to do selective multi-photon processes. However, because of the irregular structures of the pulse spectrum, some residual satellite pulses were still present in the temporal profile. Moreover, the irregular structures in the spectrum may deteriorate the ability to control multi-photon processes. In this paper, we show that in addition to phase compensation, an amplitude shaping technique can be used to smooth irregular spectral features to achieve full control of the ultrabroad-bandwidth pulses.

\section{Experiments and Results}

We used a Ti:Sapphire oscillator (Venteon Pulse 1, Nanolayers GmbH) whose ultra-broad-bandwidth spectrum can exceed $400 \mathrm{~nm}$. The output of this laser oscillator was first directed to a reflective 1:2.5 telescope and then introduced into a pulse shaper based on the general design of Weiner.[2] The pulse shaper consisted of a 150 -line-per-mm grating, a $762-\mathrm{mm}$ focal length spherical mirror and a 640-pixel dual-mask spatial light modulator (SLM)(CRI, Inc., SLM-640) and was setup in a folded geometry. The laser was focused onto a $20-\mu \mathrm{m}$ type-I KDP crystal after the pulse shaper and the second harmonic generation (SHG) signal was separated from the fundamental before it was directed to the spectrometer. (See ref [3] for details of experiment setup.)

Through the amplitude modulation, we were able to obtain the desired output spectrum. In Figure 1(a) we show the uncorrected spectrum, a rectangular and a 
Gaussian spectrum. Multi-photon intrapulse interference phase scan (MIIPS) [4, 5] was used to automatically compress the pulses and the phase distortions measured with MIIPS are also shown in Figure 1(a). Successful compensation of the spectral phase with MIIPS results in phase deviations smaller than $0.1 \mathrm{rad}$ across the spectrum (see Figure 1(a)). After phase correction, the residual phase was used to calculate the temporal profile from the amplitude-modulated spectra using Fourier transformation (Figure 1(b)). The time durations of different pulses were between 4.6-5.2 fs. One can clearly observe that Gaussian amplitude modulation resulted in almost complete elimination of the satellite pulses. Rectangular amplitude modulation reduced the time duration of the main pulse and would be very useful for selective multi-photon processes, but it did not suppress the satellite pulses.
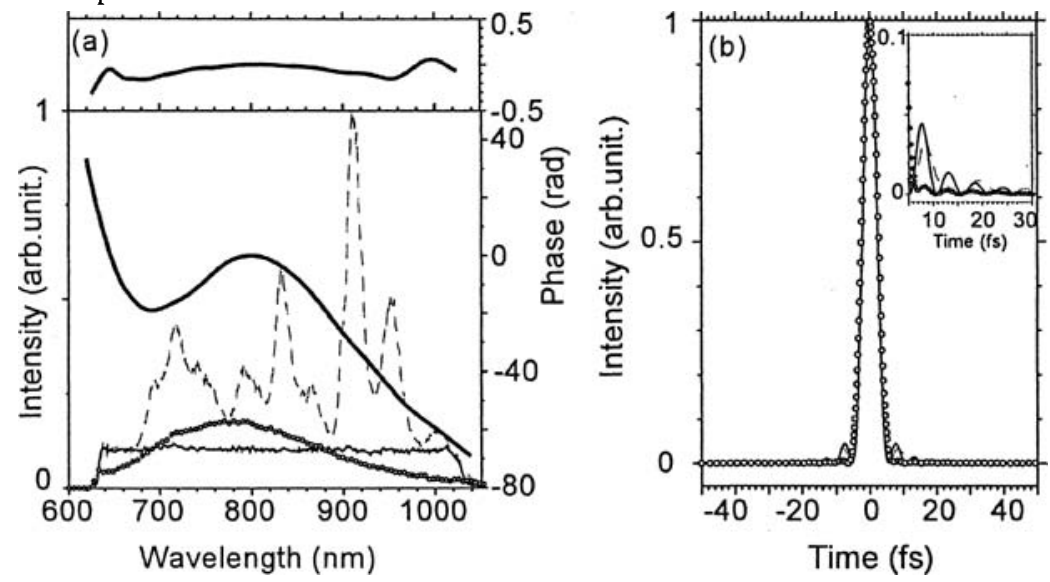

Fig. 1. (a) Spectrum and phase of the ultra-broad-bandwidth laser. The low panel shows the spectrum of the laser with (black solid and circles) and without amplitude modulation (gray dashed line). The thick lines represent the residual phase after MIIPS corrected the phase distortion (top panel) and the retrieved spectral phase of the laser system (lower panel. (b) Calculated temporal profiles for different amplitude modulations after phase correction. The gray dashed line, black solid line and circles refer to the temporal profiles for the pulses without any amplitude modulation, with rectangular amplitude modulation and with a Gaussian amplitude modulation, respectively. The inset is a closer look of the satellite pulses.

In order to demonstrate the selective control over the SHG spectrum, we introduced specific binary phases after the phase compensation. Figure 2 shows an example of SHG spectrum manipulation. The binary phase we applied contained three sections: the left section was symmetric to the center section and the right section was anti-symmetric to the center section. One can clearly see that two peaks were generated in the SHG spectrum at the positions corresponding to the symmetry points of the binary phase. This demonstrates our ability to accurately deliver ultra-broad-bandwidth shaped laser pulses. 


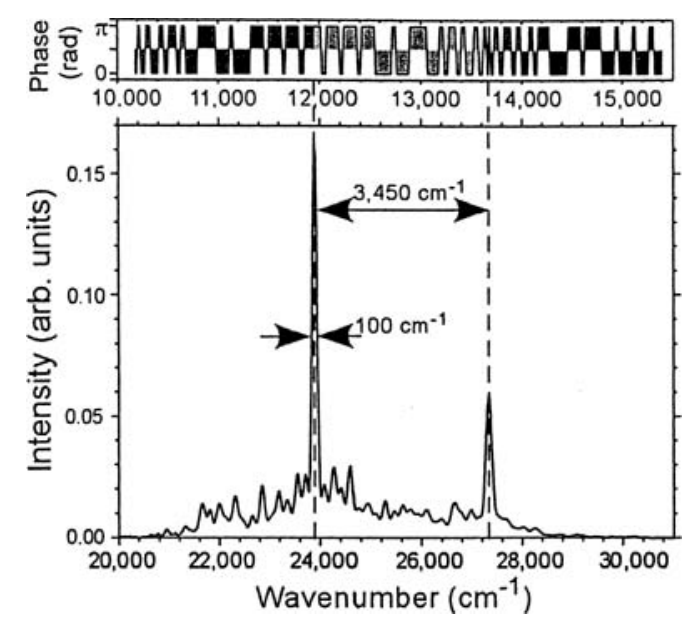

Fig. 2. Effect of binary phase shaping on second harmonic generation. Top panel shows the binary phase applied on the laser, lower panel shows the corresponding SHG spectrum.

\section{Conclusions}

In summary, we compensated an ultra-broad-bandwidth laser system whose spectrum spans over $400 \mathrm{~nm}$ using MIIPS. By correcting the amplitude as well as the phase of the laser system, we gained control of coherent pulses spanning over $400 \mathrm{~nm}$. This source is ideal for applications in CARS microscopy and selective two-photon microscopy. We also demonstrated binary phase shaping to do selective second harmonic generation.

Acknowledgements. We gratefully acknowledge support for this research by the National Science Foundation Major Research Instrumentation program.

\section{References}

1. T. Binhammer, E. Rittweger, R. Ell, F. X. Kartner, and U. Morgner, Ieee Journal of Quantum Electronics 41, 1552-1557 (2005).

2. A. M. Weiner, Review of Scientific Instruments 71, 1929-1960 (2000).

3. B. Xu, Y. Coello, V. V. Lozovoy, D. A. Harris, and M. Dantus, Optics Express (Submitted).

4. B. Xu, J. M. Gunn, J. M. Dela Cruz, V. V. Lozovoy, and M. Dantus, JOSA.B 23, 750$759(2006)$.

5. V. V. Lozovoy, I. Pastirk, and M. Dantus, Optics Letters 29, 775-777 (2004). 